\title{
Es hora de adaptar la definición de la enfermedad renal crónica de acuerdo con la edad
}

\section{It is time to adapt the definition of chronic kidney disease according to age}

\author{
John Fredy Nieto-Ríos, Mónica Zuluaga-Quintero, Edwin Jesús Ariza-Parra, \\ Diana Carolina Bello-Márquez • Medellín (Colombia) \\ laura Tatiana Gómez-Castro • Armenia (Colombia)
}

DOI: https://doi.org/10.36104/amc.2021.2080

\begin{abstract}
Resumen
La enfermedad renal crónica es una condición con alta morbilidad, mortalidad y costos elevados para los sistemas de salud que afecta todos los grupos de población con impacto significativo en su calidad de vida. Su clasificación ha sido modificada con el tiempo y aún no existe un consenso universal para diferenciar un cambio fisiológico del aclaramiento renal versus patológico. A continuación, se expone la importancia de replantear la definición y clasificación en la población general de acuerdo a la edad, incluyendo a niños y adultos. (Acta Med Colomb 2021; 46. DOI: https://doi. org/10.36104/amc.2021.2080).
\end{abstract}

Palabras clave: insuficiencia renal crónica, fallo renal crónico, clasificación, envejecimiento.

\begin{abstract}
Chronic kidney disease is a condition with high morbidity, mortality and healthcare costs which affects all population groups, having a significant impact on their quality of life. Its classification has been modified over time and there is still no universal consensus to differentiate a physiological change in kidney clearance from a pathological change. Below, we will discuss the importance of reconsidering the definition and classification in the general population according to age, including children and adults. (Acta Med Colomb 2021; 46. DOI: https://doi.org/10.36104/amc.2021.2080).

Keywords: chronic renal insufficiency, chronic renal failure, classification, aging.
\end{abstract}

Dr. Jhon Fredy Nieto-Ríos: Nefrólogo, Epidemiólogo Hospital Pablo Tobón Uribe; Dra. Mónica Zuluaga-Quintero: Internista, Epidemióloga Hospital Pablo Tobón Uribe; Dr. Edwin Jesús Ariza-Parra: Internista, Epidemiólogo. IPS Universitaria; Dra. Diana Carolina BelloMárquez: Pediatra, Epidemióloga Clínica las Américas y Hospital Infantil Concejo de Medellín. Medellín (Colombia). Dra. Laura Tatiana Gómez-Castro: Epidemióloga Clínica del Café, Dumina Medical. Armenia (Colombia).

Correspondencia: Dra. Mónica ZuluagaQuintero. Medellín (Colombia).

E-Mail: mzulu28@hotmail.com

Recibido: 19/XII/2020 Aceptado: 24/V/2021
En medicina, las definiciones y clasificaciones de las diferentes enfermedades deben ser uniformes en todo el mundo, con el fin de hacer que el personal asistencial hable el mismo idioma, lo cual promueve la detección oportuna de problemas de salud, impactando en el manejo y favoreciendo la investigación. En este artículo se analiza la importancia de replantear la definición y clasificación de la enfermedad renal crónica (ERC) en la población general de acuerdo a la edad, incluyendo a niños y adultos.

La ERC se considera un problema de salud pública con una alta morbimortalidad y costos elevados para los sistemas de salud de todo el mundo; afecta tanto a adultos y niños, con impacto negativo en la calidad de vida personal y familiar de los que la padecen. La ERC es la alteración funcional, estructural o histológica de los riñones que persiste durante tres meses o más y que tiene implicaciones en la salud del paciente. Para ello se debe cumplir que la tasa de filtración glomerular (TFG) por fórmulas validadas o en su defecto estimada, sea inferior a $60 \mathrm{ml} / \mathrm{min} / 1.73 \mathrm{~m}^{2}$ de forma persistente (mínimo tres meses), con lo cual es suficiente para clasificar al enfermo con esta patología. Si la TFG es igual o superior a $60 \mathrm{ml} / \mathrm{min} / 1.73 \mathrm{~m}^{2}$, se deben cumplir otros criterios para diagnosticar esta enfermedad tales como: evidencia de alguna alteración funcional irreversible originada en alguna de las funciones de los riñones como trastornos en la excreción de albúmina, cambios en el sedimento urinario (proteinuria o hematuria en dos de tres muestras) el control del equilibrio ácido base, electrolitos, agua corporal total, presión arterial; alteraciones estructurales como malformaciones renales, quistes patológicos, tumores, cálculos; y/o alteraciones histológicas irreversibles evidenciadas en una biopsia renal (1). 
Para realizar el diagnóstico oportuno de esta entidad, se requiere realizar un enfoque en la historia clínica completa identificando factores de riesgo, anamnesis adecuada, examen físico dirigido y herramientas diagnósticas para la detección de estas anormalidades como la medición de: creatinina, cistatina $\mathrm{C}$, nitrógeno ureico, electrolitos; uroanálisis, albuminuria; ecografía de vías urinarias, gamagrafía renal, urotomografia y/o biopsia renal de acuerdo al contexto clínico. Sin embargo, como estrategias de tamizaje en población de riesgo (identificada con una adecuada historia clínica), con análisis de orina, albuminuria y creatinina sérica es suficiente para detectar la mayoría de los casos.

A medida que progresa la ERC se presentan complicaciones como HTA, anemia, enfermedad mineral ósea, desnutrición, alteraciones electrolíticas y ácido base; estas con una alta morbimortalidad. Cuando se llega al estadio 5 , se debe plantear una terapia de reemplazo renal como la hemodiálisis, diálisis peritoneal o trasplante, y en casos seleccionados manejo conservador, todo lo cual tiene implicaciones en la calidad de vida y elevados costos para el sistema de salud (1).

Las principales etiologías de la ERC en todo el mundo son diabetes mellitus (DM), hipertensión arterial (HTA) (2), consumo de medicamentos o sustancias nefrotóxicas, glomerulopatías primarias, enfermedades autoinmunes como el lupus eritematoso sistémico (LES), infecciones urinarias en la infancia, enfermedades hereditarias renales, anomalías congénitas del riñón y el tracto urinario (CAKUT por sus siglas en inglés Congenital anomalies kidney urinary tract) $(3,4)$ etc. La DM e HTA explican aproximadamente el $80 \%$ de los casos, lo cual cobra vital importancia porque son enfermedades muy prevalentes de interés en salud pública debido a la alta morbimortalidad que tienen ya que son la principal causa de muerte a nivel mundial, puesto que favorecen problemas cardiovasculares, siendo prevenibles y tratables. Por su parte en los niños, las infecciones urinarias y las CAKUT son las principales causas de ERC, lo cual si es identificado oportunamente puede impactar favorablemente el pronóstico (2-4).

En términos económicos, la ERC se considera una enfermedad de alto costo que genera gastos directos e indirectos para el sistema de salud de Colombia, representando aproximadamente 2-4\% del presupuesto total de este. Para el 2019, se estimó 1406364 personas con diagnóstico de ERC independiente del nivel de TFG, siendo mayor el cobro para estadios 4 y 5 , en los cuales se incrementa el número de complicaciones y deterioro de la calidad de vida, sin contar los años de vida saludable y productiva que pierde una persona a causa de esta enfermedad y la alta probabilidad de morir, siendo en muchos casos temprana, debido a la mayor prevalencia de enfermedad cardiovascular en este grupo y comorbilidades asociadas como diabetes e hipertensión (5).

Desde finales de los años 90 se unificó la definición de ERC y se estableció una clasificación universal, im- pulsada por la Iniciativa para la calidad de los resultados de la enfermedad renal de la fundación nacional del riñón (NKF-KDOQI) de sus siglas en inglés, lo cual ha permitido el estudio de esta enfermedad y estandarización de los términos para hablar un lenguaje común $(1,5)$. Esta definición y clasificación se ha ido modificando a lo largo del tiempo y ha sido adoptada por gran parte de los países del mundo, incluido Colombia.

Las guías KDIGO (Kidney Disease: Improving Global Outcomes) de ERC 2012 y las guías latinoamericanas de ERC del mismo año, clasifican la enfermedad en cinco estadios que dependen de la tasa de filtración glomerular, agregándole el grado la albuminuria a cada categoría, siendo esta clasificación uniforme para todos los grupos de edad (desde niños hasta ancianos) y con una aplicación mundial. Es así como según la clasificación actual, el estadio G1 tiene una TFG $\geq 90 \mathrm{ml} / \mathrm{min} / 1.73 \mathrm{~m}^{2}$; el G2 entre 60 y $89 \mathrm{ml} / \mathrm{min} / 1.73 \mathrm{~m}^{2}$; el G3 entre 30 y $59 \mathrm{ml} / \mathrm{min} / 1.73 \mathrm{~m}^{2}$, siendo subdividido en G3a si la TFG está entre 45 y $59 \mathrm{ml} /$ $\mathrm{min} / 1.73 \mathrm{~m}^{2}$ y G3b entre 30 y $44 \mathrm{ml} / \mathrm{min} / 1.73 \mathrm{~m}^{2} \mathrm{SC}$; el G4 entre 15 y $29 \mathrm{ml} / \mathrm{min} / 1.73 \mathrm{~m}^{2}$; y el G5 menor a $15 \mathrm{ml} / \mathrm{min}$ $1.73 \mathrm{~m}^{2}$. Según la albuminuria (medida en orina como la relación albuminuria por gramo de creatinuria), la categoría A1 tiene una albuminuria inferior a $30 \mathrm{mg} / \mathrm{g}$ (denominada albuminuria normal a levemente incrementada); la A2 entre 30 y $300 \mathrm{mg} / \mathrm{g}$ (albuminuria moderada, antes conocida como microalbuminuria) y la A3 mayor de $300 \mathrm{mg} / \mathrm{g}$ (albuminuria severamente incrementada). A menor TFG y mayor albuminuria, la progresión de la enfermedad, las complicaciones asociadas y la mortalidad son mucho más elevadas $(1,6)$.

Según esta definición y clasificación, la prevalencia mundial de la ERC oscila entre 9 y $13 \%$, e incluso puede llegar al $20 \%$ en pacientes mayores de 60 años, pues existe variabilidad en los casos reportados de acuerdo con la TFG medida, el diagnóstico temprano, las enfermedades crónicas relacionadas y su registro; siendo mejor detectada en países desarrollados $(7,8)$. En Colombia, según la cuenta de alto costo para el año 2019, la ERC afectó el 1.8\% de la población, lo cual indica que hay un subregistro al compararlo con las estadísticas de otros países sumado a detección no oportuna de la ERC, donde hasta el 50\% de los casos que llegan al estadio 5 , son clasificados como de causa idiopática o desconocida (9).

El problema más grande de esta clasificación, es que sobreestima la prevalencia de la ERC en los ancianos y subestima el problema en los niños y jóvenes, debido a que no discrimina por grupo etario. Si se tiene en cuenta el envejecimiento renal fisiológico, es claro que debería haber una diferenciación de acuerdo a la edad del paciente $(10,11)$. Una persona nace con sus riñones inmaduros con un filtrado glomerular que oscila entre los 30 y $40 \mathrm{ml} /$ $\mathrm{min} / 1.73 \mathrm{~m}^{2}$, alcanzando una maduración óptima al año de edad donde se tiene una tasa de filtración glomerular que oscila entre los 90 y $120 \mathrm{ml} / \mathrm{min} / 1.73 \mathrm{~m}^{2}$ y que se mantiene hasta la adultez joven (12). A partir de los 40 años fisioló- 
gicamente se pierde entre 0.5 y $1 \mathrm{ml} / \mathrm{min}$ por año sin que esto tenga que ver con alguna enfermedad renal $(10,11)$. Para hacerlo más ilustrativo se ponen dos ejemplos:

Una mujer de 90 años, de raza blanca, sin otras comorbilidades, asintomática, con creatinina de $1.0 \mathrm{mg} / \mathrm{dl}$, con TFG calculada por BIS-1 de $43 \mathrm{ml} / \mathrm{min} / 1.73 \mathrm{~m}^{2}$ (13), con citoquímico de orina normal. Enviada a consulta de nefrología por ERC, estadio 3b A1, por alto riesgo de complicaciones, de progresión de la enfermedad, de requerir diálisis y mayor mortalidad. Si se tiene en cuenta la clasificación actual KDIGO 2012 (1), el médico remitente tiene razón y esta paciente habría sido bien clasificada. Si se basa en este simple punto de corte e igual abordaje a 100 ancianos de la misma edad, por lo demás sanos, todos quedarían clasificados de la misma forma. Sin embargo, si se tiene en cuenta que la TFG esperada a esta edad es de $40 \mathrm{ml} /$ $\mathrm{min} / 1.73 \mathrm{~m}^{2}$ (por fórmula de Keller), que está acorde con la TFG calculada (14), y que esta paciente está asintomática, con un análisis de orina normal, realmente la paciente no tiene ningún problema renal que tenga implicaciones en su salud, lo que tiene es el envejecimiento renal esperado para su edad lo cual es completamente fisiológico.

Lo anterior es un gran problema de salud pública porque hay un sobrediagnóstico de ERC en la población anciana con las implicaciones psicológicas y sociales de estigmatizar a una persona con una enfermedad de este tipo, además de los altos costos para el sistema de salud y las implicaciones de saturar el sistema de referencia, acortando de esta forma las posibilidades de atención de otros pacientes realmente afectados por esta entidad. De ahí la importancia de una adecuada definición, con el fin de reducir los recursos en salud que estarían destinados a la atención de una condición fisiológica de envejecimiento renal sin una real enfermedad renal establecida. Se aclara que, en la edad avanzada, es frecuente que se tengan múltiples comorbilidades y que finalmente una gran cantidad de ancianos tengan ERC, por esto es necesario realizar una evaluación oportuna e integral para determinar la presencia de alteración estructural, bioquímica y/o funcional renal. Sin embargo, la definición actual limita la clasificación adecuada porque no tiene en cuenta el envejecimiento renal fisiológico.

Por otro lado, en el siguiente caso, se tiene una mujer de 30 años, sin comorbilidades conocidas, a quien en un chequeo ejecutivo le documentaron una creatinina de 1.1 $\mathrm{mg} / \mathrm{dl}$ con una TFG estimada por CKD EPI de $67.3 \mathrm{ml} /$ $\mathrm{min} / 1.73 \mathrm{~m}^{2}$ verificada con la medición en orina de 24 horas y gammagrafía renal; normotensa, con examen físico normal, sin albuminuria, citoquímico de orina y ecografía sin alteraciones. Según la clasificación KDIGO de ERC este paciente no tiene ERC ya que su TFG es superior a los 60 $\mathrm{ml} / \mathrm{min} / 1.73 \mathrm{~m}^{2}$ y no tiene ningún marcador de enfermedad renal. Sin embargo, está claro que para su edad su TFG está muy por debajo de lo esperado (por fórmula de keller 100 $\mathrm{ml} / \mathrm{min} / 1.73 \mathrm{~m}^{2}$ ) (14), aunque con la evidencia disponible no hay claridad sobre la evolución de estos pacientes a largo plazo, si amerita una evaluación especializada, seguimiento y medidas de protección renal enfocadas en el estilo de vida y evitar la exposición a nefrotóxicos.

Por lo anterior, algunas autoridades mundiales en este tema, proponen una detección de la ERC basados igual en la TFG, pero teniendo en cuenta la edad del paciente. Así por ejemplo para niños y adultos jóvenes ( $<40$ años), se propone subir el límite de detección a una TFG de $75 \mathrm{ml} /$ $\min / 1.73 \mathrm{~m}^{2}$ (actualmente en 60 ), continuar en pacientes de 40-64 años con TFG límite de $60 \mathrm{ml} / \mathrm{min} / 1.73 \mathrm{~m}^{2}$ y en mayores de 65 años bajar el límite a TFG de $45 \mathrm{ml} / \mathrm{min}$, ante la falta de una clara diferencia significativa en mortalidad comparada con el punto de corte en $60 \mathrm{ml} / \mathrm{min} / 1.73 \mathrm{~m}^{2}$ en pacientes ancianos (15).

Ahora bien, en el escenario de un paciente con ERC definida por criterios funcionales, bioquímicos, anatómicos o histológicos, se mantendría la clasificación vigente según la TFG en los cinco estadios. Al igual con las categorías de albuminuria no habría cambios, como en los otros marcadores de daño renal (proteinuria, hematuria glomerular, alteración estructural o histológica). Esto permitiría evitar el sobrediagnóstico a los ancianos y el subdiagnóstico a los jóvenes, teniendo en cuenta la relación entre mortalidad y la TFG (16), con la limitante que conlleva la modificación de una clasificación ya estandarizada en todo el mundo y al discriminar por grupos se podría dificultar su aplicación ya que los médicos no especialistas en el tema podrían no recordarlo.

Finalmente, es claro que el envejecimiento renal es una condición asociada a la edad, donde la reducción de la TFG es fisiológica sin relación con las enfermedades subyacentes (16), siendo de gran impacto la detección temprana y tratamiento oportuno integral del riesgo cardiovascular y comorbilidades. Por esto, es el momento de determinar si la modificación de la definición actual a una adaptada según la edad presenta un cambio significativo en los costos en salud, afecta el seguimiento de los pacientes, genera diferencias en la mortalidad y en las complicaciones relacionadas con la progresión de la enfermedad renal. Existen diferentes posturas respecto a una correcta definición, sin embargo, es necesario lograr un consenso al respecto con el fin de acercarnos a la definición más acorde al pronóstico y aclarar las dudas relacionadas con la edad.

Como conclusiones, se resalta que la definición de ERC está unificada a nivel mundial con criterios objetivos, lo cual ha facilitado su estudio en todas las latitudes, con la limitante que no hay una distinción por grupos de edad y no se tiene en cuenta el envejecimiento renal fisiológico.

El llamado de este artículo, es a una adaptación de la definición de la ERC de acuerdo a la edad con aplicación universal, teniendo en cuenta el impacto en la morbimortalidad y calidad de vida de los pacientes, buscando estrategias que permitan optimizar los recursos de salud destinados a la atención de esta patología tan prevalente y costosa. 


\section{Referencias}

1. Levin A, Stevens PE, Bilous RW, Coresh J, De Francisco ALM, De Jong PE et al. Kidney disease: Improving global outcomes (KDIGO) CKD work group. KDIGO 2012 clinical practice guideline for the evaluation and management of chronic kidney disease. Kidney International Supplements. 2013;3(1):1-150.

2. Acuña L. Sanchez P, Soler LA, Alvis LF. Enfermedad renal en Colombia: prioridad para la gestión de riesgo. Revista Panamericana de Salud Pública. 2016;40(1):16-22.

3. Piedrahita VM, Prada MC, Vanegas JJ, Vélez C, Serna LM, Serrano AK, Flórez JA, et al. Causas de enfermedad renal crónica en niños atendidos en el Servicio de Nefrología Pediátrica del Hospital Universitario San Vicente de Paúl, de Medellín, Colombia, entre 1960 y 2010. Iatreia. 2012;24(4): 347-352.

4. Fernández C, Melgosa M. Enfermedad renal crónica en la infancia. Diagnóstico y tratamiento. Madrid Protoc Diagn Ter Pediatr.2014;1: 385-401.

5. Levey A, Eckardt KU, Dorman N, Christiansen S, Hoorn E, Ingelfinger J. Nomenclature for kidney function and disease: report of a Kidney Disease: Improving Global Outcomes (KDIGO) Consensus Conference. Kidney International. 2020;97(6):1117-1129.

6. Candelaria JC, Gutiérrez C, Bayarre HD, Acosta C, Montes de Oca DM, Labrador O. Caracterización de la enfermedad renal crónica en adultos mayores. Rev. Colomb. Nefrol. 2018;5(2):166-178. doi: http://dx.doi.org/10.22265/ acnef.0.0.308

7. Birkov B, Purcell CA, Levey AS, Smith M, Adboli A, Abebe M, et al. Global, regional, and national burden of chronic kidney disease, 1990-2017: a systematic analysis for the Global Burden of Disease Study 2017. Lancet. 2020;395(10225):709-733. doi: 10.1016/S0140-6736(20)30045-3.

8. Hill NR, Fatoba ST, Oke JL, Hirst JA, O'Callaghan CA, Lasserson DS, Et al. Global Prevalence of Chronic Kidney Disease-A Systematic Review and MetaAnalysis. PLoS One. 2016;11(7):e0158765. doi: 10.1371/journal.pone.0158765.
9. Acuña L, Valvuena A, Ramirez P, Garcia A, Ramirez N, et al. Situación de la enfermedad renal crónica, la hipertensión arterial y la diabetes mellitus en Colombia. Fondo Colombiano de Enfermedades de Alto Costo, Cuenta de Alto Costo (CAC).2019:1-293.

10. Mora JM, Slon MF, Castaño I, Izquierdo D, Arteaga J, Martínez N. Enfermedad renal crónica en el paciente anciano [Chronic kidney disease in the elderly patient]. Rev Esp Geriatr Gerontol. 2017;52(3):152-158. doi: 10.1016/j. regg.2016.03.006.

11. Bermúdez RM, Garcia SG, Rodríguez GF, Subias JE, De los Ríos MD, Melgar AA, Nieto VG. Documento de consenso: recomendaciones sobre la utilización de ecuaciones para la estimación del filtrado glomerular en niños. Anales de Pediatría. 2014;80(5):326-e1

12. Da Silva Selistre L, Rech DL, de Souza V, Iwaz J, Lemoine S, Dubourg L. Diagnostic Performance of Creatinine-Based Equations for Estimating Glomerular Filtration Rate in Adults 65 Years and Older. JAMA Intern Med. 2019;179(6):796804.

13. Keller F. Kidney Function and Age. Nephrology Dialysis Transplantation. 1987;2(5):382. doi: https://doi.org/10.1093/oxfordjournals.ndt.a091583.

14. Delanaye P, Jager KJ, Bökenkamp A, Christensson A, Dubourg L, Eriksen BO, et al. CKD: A Call for an Age-Adapted Definition. J Am Soc Nephrol. 2019;30(10):1785-1805. doi: 10.1681/ASN.2019030238.

15. Hallan SI, Matsushita K, Sang Y, Mahmoodi BK, Black C, Ishani A, et al. Chronic Kidney Disease Prognosis Consortium. Age and association of kidney measures with mortality and end-stage renal disease. JAMA. 2012;308(22):234960. doi: $10.1001 /$ jama.2012.16817

16. Eriksen BO, Palsson R, Ebert N, Melsom T, van der Giet M, Gudnason V, et al. GFR in Healthy Aging: an Individual Participant Data Meta-Analysis of Iohexol Clearance in European Population-Based Cohorts. J Am Soc Nephrol. 2020;31(7):1602-1615. doi: 10.1681/ASN.2020020151. 